\section{JOURNAL REVIEW}

\section{Randomised controlled trial of screening for Chlamydia trachomatis to prevent pelvic inflammatory disease: the POPI (prevention of pelvic infection) trial}

Oakeshott P, Kerry S, Aghaizu A, et al. BMJ 2010; 340: c1642

The prevention of pelvic infection (POPI) trial was published this year with a mixed response. Although it was a large, methodologically rigorous, randomised study involving 2529 women (mean age 20.9 years) with $94 \%$ follow-up, it was underpowered and no significant benefit of a single round of screening was demonstrated at 1 year. However, although inconclusive, the results were consistent with two previous, methodologically weaker, studies which found that screening was beneficial. The authors concluded that the effectiveness of a single chlamydia test in preventing pelvic inflammatory disease (PID) over 12 months might have been overestimated. One weakness of the study is the use of the clinical diagnosis of PID as an outcome measure.
The clinical diagnosis is likely to overestimate the diagnosis of true PID due to poor positive predictive value, while it cannot detect women with subclinical disease. Both may result in tubal disease, an important morbidity of chlamydial infection.

In POPI, women were screened at baseline and randomised to either immediate testing and treatment (5.4\% prevalence) or delayed testing and treatment $(5.9 \%$ prevalence) at 12 months. There were 38 cases of probable or possible PID, $1.3 \%(15 / 1191)$ in the intervention group and $1.9 \%(23 / 1186)$ in the control group [relative risk (RR) 0.65, 95\% confidence interval (CI) 0.34-1.22]. PID was reduced among the women chlamydia-positive at baseline (RR 0.17, 95\% CI 0.17-1.01) (1/63 in the intervention group and $7 / 74$ in the control group). Thus, in order to prevent one case of clinical PID over 12 months it may be necessary to screen 147 women. Of the women with PID who had a chlamydia result available at the time of diagnosis, $62 \%(16 / 26)$ were positive. Interestingly, 10 of the reported cases of chlamydia-positive PID were chlamydianegative at baseline. This suggests that much of PID following chlamydia infection may occur soon after infection. Thus screening every year may not be frequent enough and the authors suggested that an increased frequency of testing of women at higher risk either as a result of a recent change in partner or history of chlamydia infection in the past 3 months should be considered by policymakers.

Low and Hocking ponder about what the findings mean for the National Chlamydia Screening Programme (NCSP) in England. Should we be doing more of the same, but better? Should we be developing new approaches? Or do we need both. ${ }^{1}$ This study design is unlikely to be repeated. However, two further studies, one in The Netherlands and one in Australia that involve multiple rounds of screening and use prevalence as an endpoint, are going to provide essential additional information about the effectiveness of screening. ${ }^{1}$

Reviewed by Paddy Horner

School of Social and Community Medicine, University of Bristol, and Bristol Sexual Health Centre, University Hospitals Bristol NHS Foundation Trust, Bristol, UK

J Fam Plann Reprod Health Care 2011;37:21. doi:10.1136/fprhc.2010.0010

\section{REFERENCE}

1 Low N, Hocking J. The POPI trial: what does it mean for chlamydia control now? Sex Transm Infect 2010;86:158-159. 